\title{
Horizons in Chemical Immunology - Approaches to Synthetic Vaccine Design
}

\author{
John A. Robinson*
}

\begin{abstract}
The main aim of this short review is to provide an up-to-date perspective of the properties a synthetic molecule must have in order to elicit a pathogen-specific humoral immune response. Chemistry will have an increasingly important role to play in the future rational design and optimization of molecules that impact on the immune system, e.g. as vaccines, vaccine adjuvants, or as immunostimulants and immunosuppressants. This follows from the rapidly unfolding understanding of how the adaptive and innate arms of the immune system work, which includes the 3D structure determination of many key receptors and signalling molecules - this opens the whole field to the rational chemical design of immunomodulatory molecules (chemical immunology).
\end{abstract}

Keywords: Antibody · Antigen · Immune system · Peptide $\cdot$ Peptidomimetic

\section{What Is a Vaccine?}

The free on-line encyclopaedia (Wikipedia) defines a vaccine as:

"A vaccine is an antigenic preparation used to establish immunity to a disease. The term derives from Edward Jenner's use of cowpox ('vacca' means cow in Latin), which, when administered to humans, provided them protection against smallpox, which Pasteur and others perpetuated. The process of distributing and administrating vaccines is referred to as vaccination. Vaccines can be prophylactic (e.g. to prevent or ameliorate the effects of a future infection by any natural or 'wild' pathogen), or therapeutic (e.g. vaccines against cancer)."

\footnotetext{
${ }^{\star}$ Correspondence: Prof. J.A. Robinson Institute of Organic Chemistry

University of Zurich

Winterthurerstrasse 190

$\mathrm{CH}-8057$ Zurich

Tel.: + 41446354242

E-Mail: robinson@oci.uzh.ch
}

Exploring the definition further we are instructed that there are four types of traditional vaccine; ${ }^{[1]}$

i) Inactivated - these are previously virulent micro-organisms (including viruses) that have been killed with chemicals or heat. Examples are vaccines against flu, cholera, bubonic plague, and hepatitis A;

ii) Live, attenuated - these are live microorganisms that have been cultivated under conditions that disable their virulent properties. Examples include yellow fever, measles, rubella, and mumps;

iii) Toxoids - these are inactivated toxic compounds from micro-organisms in cases where these (rather than the micro-organism itself) cause illness. Examples of toxoid-based vaccines include tetanus and diphtheria;

iv) Subunit - rather than introducing a whole inactivated or attenuated micro-organism to an immune system, a fragment of it can create an immune response. A characteristic example is the subunit vaccine against hepatitis B that is composed of only the surface proteins of the virus (produced in yeast).

There can be little doubt that such vaccines have contributed enormously to global human health for well over a century. ${ }^{[2,3]}$ However, it is also true that these successful vaccines were developed without any real molecular understanding about how the immune system works, nor what it takes to design an effective vaccine. ${ }^{[4]}$ The term alchemy might be appropriate to describe this trial-and-error ap- proach to transmuting potencies, if it were not for the fact that modern 'chemistry' had very little to do with it. Even today, most commercial vaccines are based on whole or inactivated organisms. ${ }^{[1]}$ An important message of this article, however, is that chemistry will have an increasingly important role to play in the future rational design and optimization of molecules that impact on the immune system, e.g. as vaccines, vaccine adjuvants, or as immunostimulants and immunosuppressants.

\section{The Age of Reverse Vaccinology}

In recent years genomic information has been used extensively in subunit vaccine design. ${ }^{[3,5-7]}$ Rather than relying on Pasteur's approach of laboriously attempting to isolate, inactivate and test the infectious agent, or a component of it, the finished genome sequence can now be mined for potential antigens that can be produced and tested in the form of recombinant proteins - an approach called 'reverse vaccinology'. ${ }^{[8]}$ However, although hundreds of potential protein antigens from a single organism can be identified, produced and tested, almost in a high-throughput mode, the approach is fraught with many problems. For example, it is usually not clear whether the proteins produced are properly folded. It is also often not clear whether protective epitopes are present, i.e. protein surface patches that can be bound by antibodies that inhibit the life cycle of the infectious agent. The whole surface of a protein may be immunogenic, 
but typically many of the antibodies that are elicited against a protein may have no deleterious effect on the life cycle of the pathogen. In some cases, the bound antibodies may even promote the life cycle of the pathogen, or after binding, may block the actions of other antibodies that do have inhibitory activity.

An alternative approach, which has also been termed 'reverse vaccinology', ${ }^{\text {9] }}$ involves first identifying the key epitopes on selected target antigens, which are bound by neutralizing or inhibitory antibodies, and using this information as a basis for vaccine design. For example, antibodies might be isolated from infected humans and tested for their ability to bind the target and inhibit infection. Ultimately, a 3D crystal structure of the inhibitory antibody (or the Fab fragment) bound to the target protein would reveal where the epitope is located, and its bound structure. This structural information then provides a starting point for the design of epitope mimetics as potential vaccine candidates. The precise characterization of the surface epitopes recognized by neutralizing antibodies is the first step in a rational chemical approach to creating polypeptides, peptidomimetics, or other small molecules, that mimic inhibitory epitopes. Through 'chemistry' it may be possible to stabilize in smaller peptides or peptidomimetics the conformations important for antibody recognition. Examples can be seen in recent efforts to design immunogens that can elicit broadly neutralizing antibodies to gp120 on HIV-1..10]

\section{Synthetic Peptides as Vaccines}

A great interest within the biological community was generated in the 1980s through the observation that sometimes a small linear peptide fragment of a protein could be used to elicit antibodies in an animal that would cross-react with the cognate native protein antigen on the surface of a parasite. This interest was marked in 1986 by a CIBA Foundation symposium. ${ }^{[11]}$ The potential importance of this observation was very great. For instance, by using such peptides as antigens, it might be possible to focus the (humoral) immune response on just those protein epitopes that are recognized by inhibitory antibodies. Also, these small protein fragments could be made through chemical synthesis. This could potentially avoid many of the problems encountered in producing recombinant proteins, correctly folded, with the levels of characterization and purity required for clinical use. However, it must now be said that there are currently no commercial synthetic peptide vaccines, against any infectious agent, in human clinical use. Several key reasons why this approach has so far

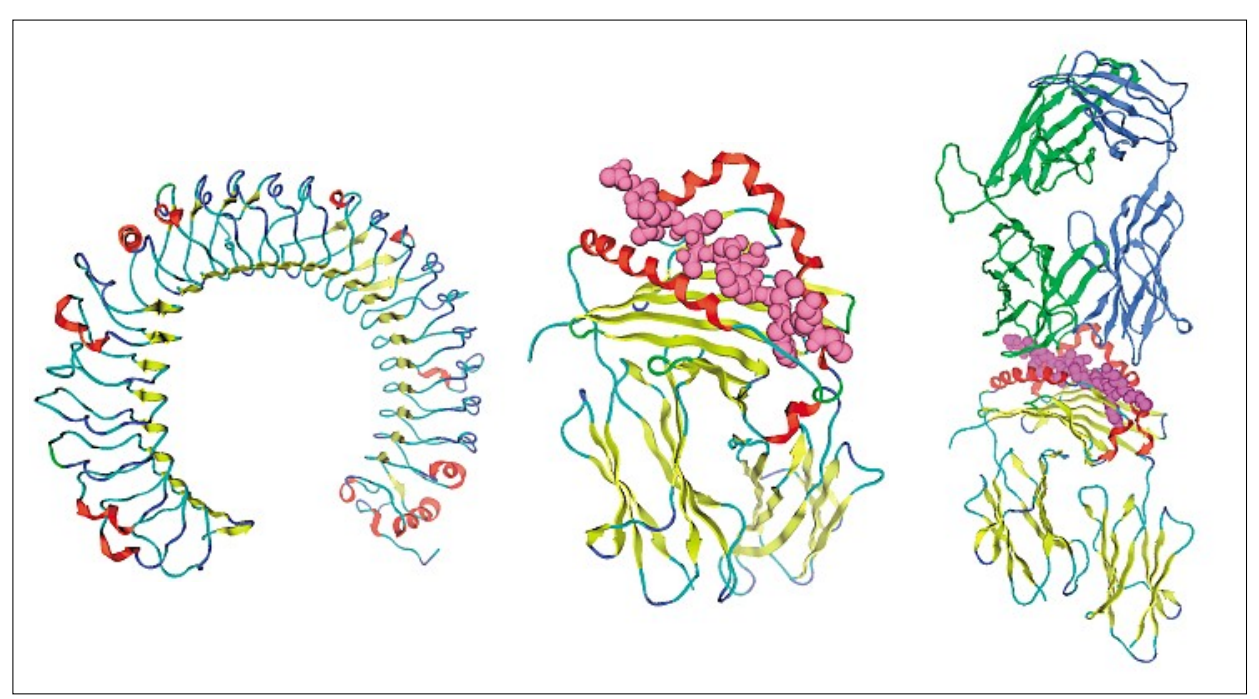

Fig. 1. Ribbon representations of X-ray crystal structures of the extracellular domain of Toll-like receptor-3 (TLR-3) (left, PDB 1ZIW); one (of the many genetically diverse) MHC class-II receptors with a bound peptide (middle, the bound peptide is shown in purple as a CPK model, PDB 1DLH); and one example of a T cell receptor domain bound to a MHC-II - peptide antigen complex (right, TCR at the top, peptide-MHC-II at the bottom, PDB $1 \mathrm{~J} 8 \mathrm{H}$ )

not been translated into successful vaccine products can be quickly recognized.

One key reason why peptides (and synthetic molecules in general) have so far not fared well as vaccine candidates is related to the fact that small synthetic molecules are generally only very poorly immunogenic, and require specialized methods of delivery. The immune systems of humans and other animals did not evolve to resist assaults from synthetic organic chemists, but rather from invading viruses, bacteria, parasites and other infectious agents. ${ }^{[12]}$ Many organisms have evolved mechanisms to detect the presence in the body of pathogens, and to provide an initial response in the fight against them, before the adaptive immune system kicks-in. This involves the so-called innate immune response, ${ }^{[13]}$ which does not make use of libraries of antibodies and T-cell receptors generated through gene shuffling and somatic mutation, but rather is based on specific germ-line encoded receptors and signalling pathways, and employs the activation of dendritic and natural killer cells, the tagging of antigens by proteins of the complement system, and in a first line of defence the release of anti-infective agents, e.g. antimicrobial peptides, produced in the body.

\section{The Innate Immune Response - Sniffing out PAMPS with PRRs}

The innate immune response against infectious agents begins by the recognition of specific structures (molecules) uniquely present in, or on, the invading organism. ${ }^{[14,15]}$ These are often called pathogen-associated molecular patterns (PAMPs) ${ }^{[16,17]}$ (a fancy name for molecules like lipopolysaccharide, viral RNA or DNA, bacterial lipopeptides, and others), which are foreign to the host animal but an integral part of the pathogen (bacterium, virus, parasite etc.). These PAMPs are recognized by specific membrane-bound pattern recognition receptors (PRRs), including over a dozen different Toll-like receptors (TLRs), expressed on the sentinel cells guarding the peripheral tissues, in particular, macrophages and dendritic cells. ${ }^{[18-20]}$ Dendritic cells are key sentinels in the periphery, where they sniff-out (through their TLRs), and then capture and process antigens. ${ }^{[21-24]}$ Very recently, the first X-ray structure of the extracellular portion of TLR-3 was reported (Fig. 1), which revealed not only a remarkable protein architecture containing multiple leucine rich repeats, but also provided clues as to how the receptor binds its ligand, in this case viral RNA. ${ }^{[25-26]}$ Already work has begun to design novel small drug-like molecules that bind to specific TLRs and so can modulate immune responses ${ }^{[14-16]}$ (see below). For example, synthetic cytosine-phosphate-2'-deoxy-7-deazaguanosine dinucleotides (CpR) (Fig. 2), called immunomodulatory oligonucleotides (IMOs) have been described that induce strong and rapid immunostimulation through binding to TLR-9, thus leading to immune-cell activation and cytokine secretion in vitro and in vivo. ${ }^{[27,28]}$

More importantly, however, the recognition of PAMPs by TLRs and other PRRs at the cell surface, triggers intracellular signalling pathways, which culminate in the maturation of dendritic cells and the release of pro-inflammatory cytokines, chemokines, and type-I interferon. The conversion of immature to mature dendritic cells is accompanied by a marked cellu- 


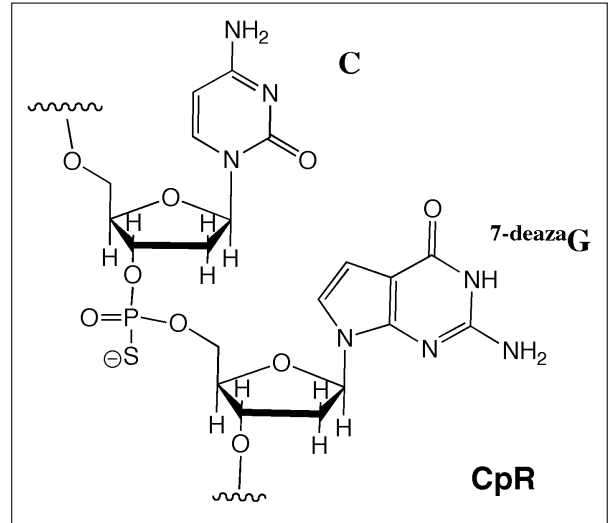

Fig. 2. Synthetic cytosine-phosphate-2'-deoxy7-deazaguanosine dinucleotides (CpR) as TLR-9 agonists and potent immunomodulators

lar reorganization, including the redistribution of MHC class-II molecules to the cell surface (Fig. 3), which is important in the next step, the activation of $\mathrm{T}$ helper cells ${ }^{[21]}$ (see below). This is all rather like sending up smoke signals, the end result being that the cavalry (the $\mathrm{B}$ and $\mathrm{T}$ cells in the adaptive immune system) are alerted that something is wrong, which requires their attention. Of course, typical synthetic organic molecules (including most small peptides) do not elicit this response. For this reason, when synthetic molecules are tested as vaccines, they are usually linked to a carrier protein and co-injected with an adjuvant that can. Adjuvants are (simply put) components added to vaccine formulations that enhance the immunogenicity of antigens in vivo. Excluded from this definition would be delivery vehicles that allow multivalent display of antigens (see below), targeting of antigens to antigen-presenting cells, and concentrating and co-localizing antigens with immune potentiators (e.g. TLR agonists). The design of new adjuvants is an active area of research. ${ }^{[14,29,30]}$ Perhaps the best known adjuvant is Freund's complete adjuvant. This is a rather obnoxious cocktail comprising a suspension of desiccated mycobacterial cells in a mixture of paraffin oil and an emulsifying agent, mannide monooleate. Injecting this under the skin elicits a strong general pro-inflammatory immune response that can even cause the death of laboratory animals, and so cannot be used in humans. There are presently very few effective adjuvants licensed for human use, one of the most widely used being alum $^{[31]}$ (antigens absorbed onto aluminium and calcium salts, to produce a particulate preparation with mild immunostimulatory properties). Another adjuvant (called AS02A) developed by GlaxoSmithKline Biologicals, and used recently in clinical tests of a malaria vaccine, ${ }^{[32]}$ comprises a proprietary oil-in-water emulsion containing the immunostimulants 3-deacylated monophosphoryl lipid $\mathrm{A}$ and a triterpene saponin mixture (QS21) isolated from the plant Quillaja saponaria. ${ }^{[33]}$ Apart from the design of the antigen, one of the key problems in vaccine discovery for human use lies in finding an effective but harmless adjuvant, or some alternative way of eliciting an efficient immune response. ${ }^{[14]}$ Alternative approaches to antigen delivery are now being explored that do not require adjuvants, and which exploit the growing knowledge of the mechanisms through which the adaptive immune system is activated in the body.

We should also not forget that a second key component of the immune response to an infectious agent, apart from the antibody response, is based on the activation of CD8 ${ }^{+}$cytotoxic $\mathrm{T}$ cells and natural killer cells whose job it is to identify and destroy cells of the host that have become infected with pathogens. The activation of $T$ cells occurs when the $\mathrm{T}$ cell receptor recognizes a linear peptide fragment derived from the invading pathogen, and bound in the groove of the MHC class-I receptor, on the cell surface. The peptide-MHC class-I complex is a marker, which serves to single out the infected cell for destruction by activated $\mathrm{T}$ lymphocytes and natural killer cells (for a video animation see: http:// www.serotec.com/FlashAni/flash_animation_landing.htm? =science). This process will not be discussed further, since the main focus here is on the process of B cell activation leading to a humoral (antibody) immune response.

\section{B Cell Activation}

The rapid production of antibodies is crucial to most anti-pathogen immune responses. This requires the activation of antigen specific naïve $B$ cells and T helper cells. The activated $\mathrm{B}$ cells receive help from cognate $\mathrm{CD}^{+}{ }^{+} \mathrm{T}$ helper cells, and eventually become the antibody factories (plasma B cells) that secrete large amounts of the specific and optimized antibody. Yet $\mathrm{B}$ and $\mathrm{T}$ cells that specifically recognize one particular antigen are exceedingly rare in the body, so how are they activated and how do they find each other? Although only partly understood, it is clear that näive $\mathrm{B}$ and $\mathrm{T}$ cells (i.e. ones that have not previously been activated by antigen) get together with antigens within the lymphoid organs (e.g. the lymph nodes and spleen). The lymph nodes constitute, if you like, the 'mating bars' where the players (antigen and näive $B$ and $T$ cells) gather and survey each other. Recirculating naïve B cells enter secondary lymphoid organs continually from the blood to scan for their specific antigens. So clearly antigens must be transported from the site of entry (e.g. a splinter in your finger, or an injection under the skin) through the lymphatic system to the lymph nodes, where they can meet up with prospective naïve B and T cells.

Antigens appear to arrive in lymph nodes as soluble antigen, as immune complexes, or in association with antigen presenting cells (APCs) e.g. dendritic cells. ${ }^{[34,35]}$ Some small model antigens such as hen egg lysozyme appear to gain ready access to lymphoid follicles, but others, particularly those of larger sizes or associated with particulate materials, seem to be transported into follicles by antigen transport cells. Details of how this occurs are still murky. But dendritic cells activated in the epithelial surfaces (i.e. that have seen the smoke signals) can transport

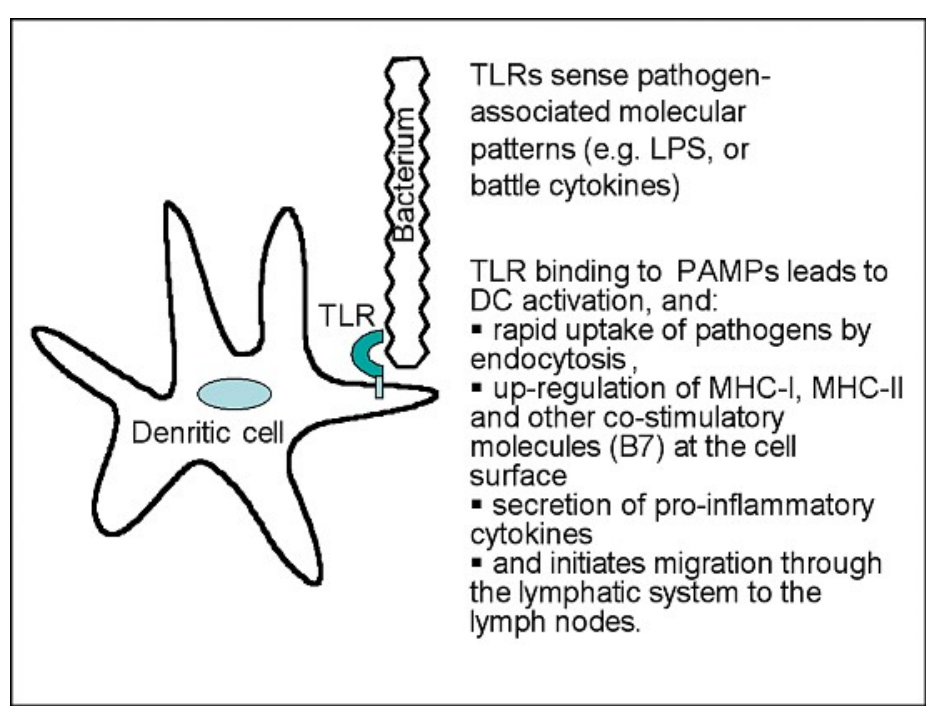

Fig. 3. Dendritic cells (DCs) are white blood cells, produced in the bone marrow, which take up sentinel positions in the tissues. An important function is to present antigen fragments to $\mathrm{T}$ cells via $\mathrm{MHC}$ molecules in the lymph nodes. 
large amounts of engulfed antigen, in an intact form, to lymphoid organs for scrutiny by $\mathrm{B}$ and $\mathrm{T}$ cells. ${ }^{[36]}$ In addition, antigen that has been opsonized (decorated) by complement or antibodies can be carried by the lymph into the node. Within the lymph nodes, follicular dendritic cells (FDCs), dendritic cells from the periphery, and macrophages can display intact antigen, held on their cell surface, to naïve $B$ cells during the initial stages of a primary response. The FDCs can capture antigen opsonized by complement or antibodies, using complement receptors and Fc-receptors on theircell surface. TheB-cell receptors (BCRs) on the surfaces of naïve $B$ cells can then screen the presented antigen until an optimal fit is obtained, and the $B$ cell is then activated.

It has long been known that the immune system generates robust immune responses against pathogens displaying repeated antigenic structures across a surface, as found for example on a virus or bacterium. ${ }^{[12,37]}$ In particular, B cells seem to be most effectively activated by membranebound antigens. ${ }^{\left[{ }^{[8]}\right.}$ Recently, new insights have been reported into how B cells recognize and are activated by antigens. The $\mathrm{BCR}$ is a multiprotein complex comprising a membrane-bound antibody molecule that is non-covalently associated with an $\operatorname{Ig} \alpha / \operatorname{Ig} \beta$ heterodimer (Fig. 4). Engagement of repeated antigenic structures by multiple BCRs on the surface of the B cell, leads to a two-phase response, in which the B cell first spreads over the antigen-bearing membrane and then contracts, thereby collecting bound antigen into a central aggregate. ${ }^{[39]}$ The extent of this response determines the quantity of antigen accumulated and hence the degree of $B$ cell activation. Only if both the antigen affinity and density is high enough, to enable successive engagement with multiple BCRs, does the initial spreading and then contraction continue, which leads finally to formation of an immunological synapse between the two cells ${ }^{[38]}$ (the term immunological synapse was first introduced to describe the contacts made between TCRs and peptide-MHC complexes on APCs, during $\mathrm{T}$ cell activation, see below). Following synapse formation, the antigenBCR complex will be internalized by the $\mathrm{B}$ cell and enter the normal MHC processing pathway. ${ }^{[40,41]}$

\section{Activating T Helper Cells}

A second and more important reason why small molecules are often coupled to carrier proteins in order to raise an immune response, is to ensure the activation also of cognate $\mathrm{T}$ helper cells. $\mathrm{T}$ lymphocytes ( $\mathrm{T}$ cells) are so called because of the role that

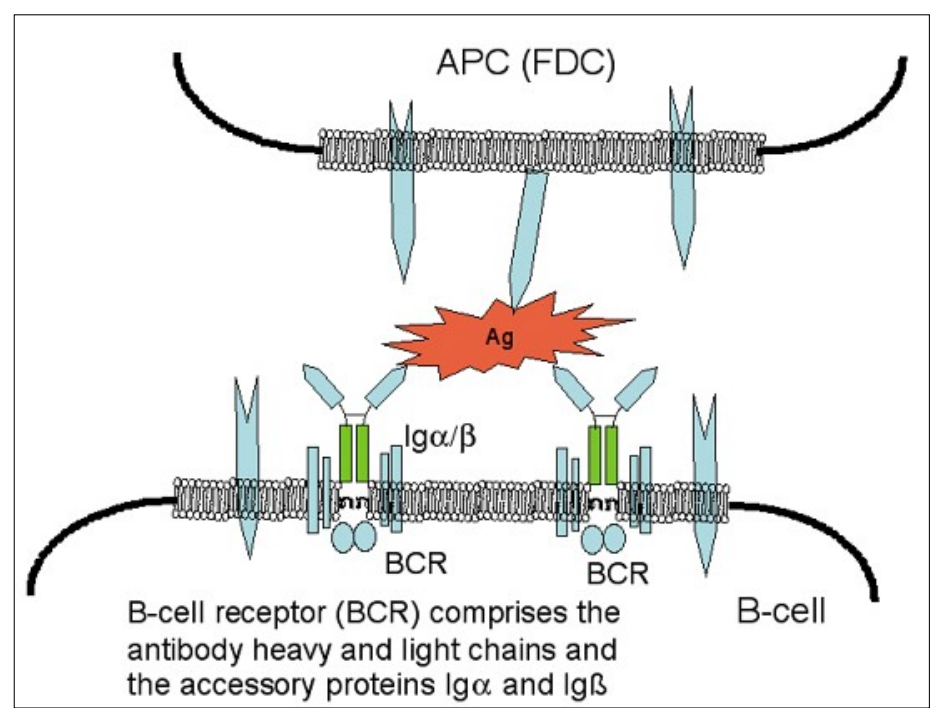

Fig. 4. Follicular dendritic cells (FDCs) in the lymph node display antigen to $B$ cells. FDCs resident in lymph nodes (not the same as the peripheral DCs) may capture antigen that has been bound (opsonized) by antibodies or complement, via the Fc-receptor or complement receptor, respectively. Multiple BCRs can be cross-linked by binding to repeated epitopes on the antigen. Other adhesion molecules on each cell surface further strengthen the contact, leading to an immunological synapse, ${ }^{[38]}$ cell activation, and BCR-antigen internalization

the thymus gland plays in their development (in particular, elimination of self-reactive $\mathrm{T}$ cells). In general $\mathrm{T}$ cells, unlike $\mathrm{B}$ cells, do not recognise intact antigen, but rather require the protein antigen to be cleaved into peptides by proteolysis within the cell. Peptide fragments can then be loaded onto class I or class II major histocompatibility complex (MHC) molecules (Fig. 1), and the complexes can be presented on the cell surface, ready for scrutiny by $\mathrm{T}$ cells. In general, MHC-I receptors (which are present on most cells in the body) present antigen to $\mathrm{CD}^{+} \mathrm{T}$ cells, whereas MHC-II receptors (which are only found on antigen presenting cells (APCs)) present antigen to $\mathrm{CD}^{+}{ }^{+} \mathrm{T}$ cells. There are distinct intracellular pathways of antigen processing for presentation with either class I or class II MHC molecules. Antigens for presentation in association with MHC-I molecules are derived from the cell's cytosol, whereas the majority of antigens presented by MHC-II molecules are derived from antigens taken up from outside the cell and processed in cellular compartments such as endosomes.

Expression of a unique T-cell receptor (TCR) confers antigen specificity to a specific $\mathrm{T}$ cell. The structure of the TCR is distinct from the antigen receptor on $\mathrm{B}$ lymphocytes (BCR), but a huge diversity of TCRs is achieved in the pool of T lymphocytes in the body by a process of TCR gene rearrangements similar to the rearrangement of $\mathrm{V}, \mathrm{D}$ and $\mathrm{J}$ segments of immunoglobulin genes seen in B cells. Furthermore, $\mathrm{T}$ cells express either the CD4 or the CD8 surface protein, and these markers can be used to define two major sub-popula- tions of mature $\mathrm{T}$ cells in the periphery. The $\mathrm{CD}^{+} \mathrm{T}$ lymphocytes represent the 'helper' $\mathrm{T}$ cell population. The $\mathrm{CD} 8^{+} \mathrm{T}$ lymphocytes represent the antigen specific cytotoxic $\mathrm{T}$ lymphocytes (CTL), which respond to and kill cells that are infected with intracellular pathogens, such as viruses, some intracellular bacteria (e.g. Listeria) and some intracellular protozoa (e.g. malaria parasites). CD8 specifically recognizes and binds MHC-I, whereas CD4 binds to MHC-II. These interactions strengthen the adhesion between the APC and the T cell.

CD4+ $\mathrm{T}$ lymphocytes are often known as 'helper' T cells, as they act on other cells of the immune system, in particular B cells, to promote $\mathrm{B}$ cell maturation by stimulating immunoglobulin isotype switching (e.g. change from an initial $\operatorname{IgM}$ to $\operatorname{IgG}$ or $\operatorname{IgE}$ ) and affinity maturation of the antibody response. Naïve $\mathrm{CD}^{+}{ }^{+} \mathrm{T}$ cells use their TCRs to search for a complementary peptide antigen in a complex with a class II MHC molecule (Fig. 1), on the surface of APCs (B cells, macrophages and dendritic cells) (Fig. 5). The TCRs on T cells scan peptideMHC-II complexes on professional APCs that have taken up and processed the protein antigen. Once a CD4+ $\mathrm{T}$ cell has found its complementary target peptide-MHC complex, a second co-stimulatory signal must be sent from the APC to the T cell. This is provided by specific interactions between other co-stimulatory cell surface markers (e.g. B7 proteins on APCs, which bind CD28 on T cells). During activation, a clustering of TCRs engaged with peptideMHC complexes is found, surrounded by complexes formed by other adhesion mol- 
ecules at the point of contact between the $\mathrm{T}$ cell and APC. This results in the formation of a so-called 'immunological synapse'. ${ }^{[42-44]}$ Recent imaging analyses have revealed that TCR microclusters, formed prior to synapse formation, are the site for antigen recognition and $\mathrm{T}$ cell activation. ${ }^{[22-24]}$ The interface, although quite dynamic, can be stable for hours. ${ }^{45,46]}$ Only a few co-stimulatory molecules seem to be involved in initial $\mathrm{T}$ cell activation. However, the picture becomes more complicated for re-stimulation of T cells that have already 'seen' the antigen. The mechanism of naïve $\mathrm{T}$ cell activation in central lymphoid organs seem to be different to the co-stimulation of antigen-experienced $\mathrm{T}$ cells, which may occur in the lymphoid organs and also in the periphery. ${ }^{[47]}$

\section{T and B Cell Proliferation}

After activation, the cells disengage and the $\mathrm{T}$ helper cell proliferates, while the APC goes on to activate other T cells. One consequence of $\mathrm{T}$ cell activation is the upregulation of another surface marker on $\mathrm{T}$ cells, namely CD40L. This is important for further stimulation of APCs, and for B cell activation. The $\mathrm{B}$ cell, namely, requires a second signal before it is activated, and unleashes its antibody production facility. The first signal is provided by BCR engagement with antigen. A second signal is provided by the interaction of CD40 on the B cell with CD40L on the surface of activated T helper cells (Fig. 5) (i.e. the B and T cells have to agree that an invader is at hand before triggering an immune reaction). This is not an antigen specific interaction, but is essential for the so-called T cell-dependent immune response. A T-cell independent antibody response can also occur, in particular, when repeated epitopes (e.g. carbohydrates) on a pathogen cross-link a large number of BCRs. Carbohydrates elicit T-cell independent responses, and in such cases a second activation signal is provided, not by the T cell, but by cytokines secreted by dendritic cells after activation.

$\mathrm{CD}^{+}{ }^{+} \mathrm{T}$ cells act by releasing cytokines in response to antigenic stimulation. $\mathrm{Cy}-$ tokines are soluble intercellular messenger molecules, which interact with specific receptor molecules on target cells. By releasing cytokines in response to antigenic stimulation, $\mathrm{CD}^{+}{ }^{+} \mathrm{T}$ cells are able to orchestrate an appropriate cell-mediated immune response to infection. Helper T cells can be divided into two broad groups, based on the specific profiles of cytokines they release. These groups are known as Th1 and Th 2 cells. Th1 cells release predominantly IL-2, TNF and IFN $\gamma$ in response to antigenic stimulation; IFN $\gamma$ primes macrophages and influences B cells during class

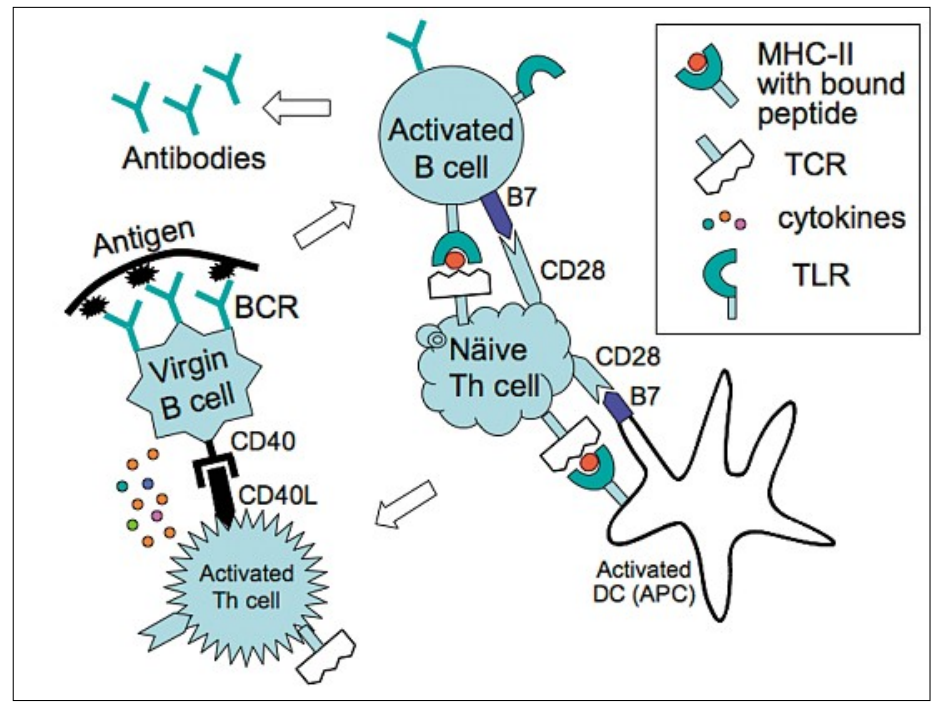

Fig. 5. Summary (and simplification) of some of the key interactions during $\mathrm{B}$ and $\mathrm{T}$ helper cell activation

switching to produce IgG3 antibodies; TNF activates primed macrophages and natural killer cells and IL-2 promotes immune cell proliferation - the perfect package to help defend against a viral or bacterial attack in the blood and tissues. Th2 cells secrete IL4, IL-5 and IL-10; IL-4 is a growth factor for B cells and influences class switching to produce IgE antibodies; IL-5 is a cytokine that promotes class switching to $\operatorname{IgA}$; and IL-10 acts to decrease the rate of proliferation of Th1 cells - a cocktail that is ideal to defend against parasitic (IgE) or mucosal (IgA) infection, whilst skewing the immune response towards an 'allergic' type of reaction. Actually, rather than viewing helper $\mathrm{T}$ cells as falling into two distinct groups, it is probably more accurate to view them as forming a continuous spectrum, with Th1 and Th2 cells at opposite ends of the scale, and the so-called Th0 cells remaining unbiased and being able to produce a wide range of cytokines. Th1/Th2 profiles represent local responses to deal with local infections in the body. But how do the T cells know what type of profile to produce, and where in the body to release it? Here again the innate immune system plays a key role. The dendritic cells in the periphery use their PRRs to sniff out the PAMPs. The dozen or more different TLRs on dendritic cells each recognize different molecular patterns characteristic of the broad classes of invaders, e.g. TLR-4 detects bacterial products, TLR-3 senses viral RNA etc. Also, different areas of the body produce characteristic mixtures of cytokines in response to invaders. This information is somehow recorded in the different co-stimulatory molecules expressed on the surface of the dendritic cells, and when these cells migrate to the lymph nodes to activate $\mathrm{T}$ cells, this information is 'read' by co-receptors on the $\mathrm{T}$ helper cell surface. ${ }^{[23,45,47]}$

\section{The Three Signals in B Cell Activation}

A further advance in understanding the mechanism(s) of B cell activation was made recently. This activation process is kept under strict control, in order to avoid release of auto-reactive and potentially pathogenic lymphocytes. As outlined above, for activation the B cell needs to receive two signals, one through cross-linking of BCRs with antigen at the cell surface, and the second through interaction with an activated $\mathrm{T}$ helper cell. Recent evidence, however, has shown that efficient B cell priming and the launch of a $\mathrm{T}$ cell-dependent immune response is stimulated by a third signal, namely, the direct activation of TLRs on B cells. ${ }^{[48,49]}$ TLRs on dendritic cells are important for detecting pathogen-associated molecular patterns (PAMPs) during the process of $\mathrm{T}$ helper cell maturation. They seem, however, to be used again in the same way during $B$ cell activation. Thus naïve human $B$ cells express low to undetectable levels of TLRs, but BCR cross-linking by antigen leads to rapid up-regulation of TLR expression. The B cells are then able to respond directly to molecules that interact with TLRs (Fig. 5). Thus, naïve B cells are activated in response to all three signals much more effectively than in response to BCR-cross linking and $\mathrm{T}$ helper cell priming alone. This has the interesting consequence that synthetic agonist ligands of TLRs have the property of boosting significantly primary B cell responses.

Synthetic $\mathrm{CpG}$ oligonucleotides, for example (Fig. 2), that are ligands for TLR-9 synergize with $\mathrm{T}$ cell help and BCR cross-linking to induce primary $\mathrm{B}$ cell responses. ${ }^{[27,28,49]}$ Other synthetic molecules that behave in a similar way include various derivatives of lipopeptides 
such as tripalmitoyl-S-glyceryl cysteine (N-palmitoyl-S-(2,3-bis-(O-palmitoyloxy)-propyl)-cysteinyl- or Pam3Cys) and dipalmitoyl-S-glyceryl cysteine (2,3-bis(O-palmitoyloxy)-propyl)-cysteinyl- or Pam2Cys) ${ }^{[50,51]}$ (Fig. 6). These lipid moieties are found in lipoprotein components of the inner and outer membranes of gramnegative bacteria. Synthetic lipopeptides carrying these or related di-acylated or triacylated S-glyceryl cysteine residues at the $\mathrm{N}$-terminus have been shown to be specific ligands of TLRs, ${ }^{[52]}$ and the conjugation of peptide antigens to Pam3Cys or Pam2Cys has been applied in the design of self-adjuvanting synthetic vaccine candidates. ${ }^{[53-55]}$ Another example is seen in the synthetic malaria vaccine candidate shown in Fig. 7 , which has recently been tested in the clinic. ${ }^{56,57]}$ This molecule is based on the MAP technology (see later), and combines $\mathrm{B}$ and $\mathrm{T}$ cell epitopes derived from the NPNA-repeat region of the circumsporozoite (CS) protein in Plasmodium falciparum, together with a universal $\mathrm{T}$ helper cell epitope also derived from the CS protein, and the Pam3Cys immunostimulatory lipid. It seems certain that there are many other

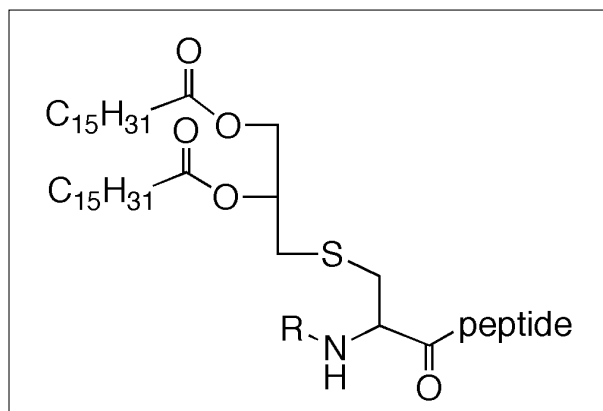

$$
\begin{array}{ll}
R=H & \text { Pam }_{2} \text {-Cys- } \\
R=\text { palmitoyl } & \text { Pam } \\
\text {-Cys- }
\end{array}
$$

Fig. 6. Lipopeptides such as tripalmitoyl-Sglyceryl cysteine (N-palmitoyl-S-(2,3-bis-(Opalmitoyloxy)-propyl)-cysteinyl- or Pam3Cys) and dipalmitoyl-S-glyceryl cysteine (2,3-bis-(Opalmitoyloxy)-propyl)-cysteinyl- or Pam2Cys) have potent immunostimulatory effects avenues for the discovery and application of novel pathogen-derived immunomodulatory molecules. ${ }^{[16]}$

\section{B Cell Maturation}

B cells express new proteins on their surface after activation, including the IL-2receptor, whose ligand, IL-2, stimulates B cells to proliferate. The major suppliers of IL-2 and other cytokines are T helper cells, so $\mathrm{T}$ cell help is usually required for a clone of 'selected' B cells to be produced. Following this, antibody class switching and affinity maturation of the selected antibody occurs, which are controlled by cytokines that $\mathrm{B}$ cells encounter when switching takes place. Thus, once activated, B cells first start to produce IgM, which are very good at activating the complement cascade. This is an excellent first line of defence against invading viruses or bacteria. Class switching from IgM to one of the other antibody classes ( $\operatorname{IgG}, \operatorname{Ig}$ A or IgE), occurs simply by exchanging the genetic information for the constant regions of the antibody heavy chain; the part of the antibody that binds to the antigen (the variable domains) is not altered. Through class switching, new classes of antibodies are generated that are better equipped to combat certain types of pathogen, e.g. IgA for combating infections in the mucosal surfaces, IgG for fighting viruses and bacteria in the circulation and periphery, $\operatorname{IgE}$ for defending against parasites.

Somatic hypermutation of the antibody variable regions, and selection for antibodies having higher affinity for the antigen occur usually after class switching. B cells that still make IgM usually have not undergone somatic hypermutation. This includes $\mathrm{B}$ cells activated without $\mathrm{T}$ cell help, e.g. in response to carbohydrates, which usually do not class switch or undergo somatic hypermutation, and so are typically of low affinity. For maturing B cells to continue to proliferate and undergo somatic hypermutation they must be continually re-stimulated, by binding to antigen and receiving $\mathrm{T}$ cell

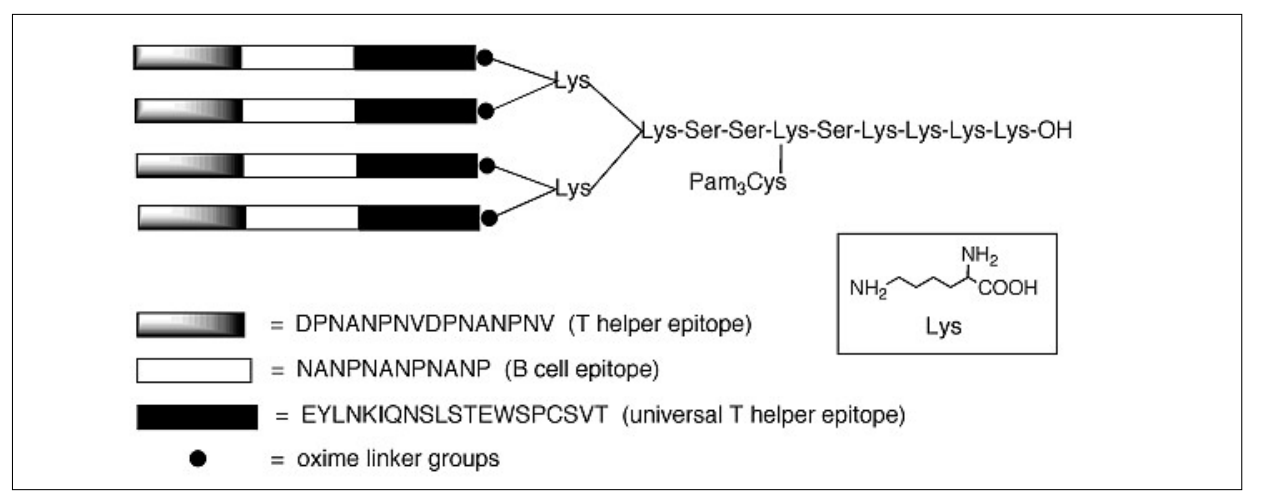

Fig. 7. A synthetic malaria vaccine candidate based on MAP technology, including universal Thelper epitope and B cell epitopes ${ }^{[56,57]}$ help. The activation of B cells leads to upregulation of MHC-II and co-stimulatory molecules on the cell surface, so the B cell can also function as an APC to restimulate T helper cells (Fig. 5). B cells are not used as APCs in the initial stages of infection when they are still naïve. Later during infection, or upon re-infection, they can act as APCs, where importantly, their improved affinity for the antigen helps to concentrate the antigen for processing in the cell and presentation on the surface with MHC-II molecules. $B$ cells have a huge advantage over other APCs in activating helper $T$ cells at times when there is little antigen around.

The importance of this last point becomes clearer by noting that the secondary lymphoid organs have segregated areas where näive $\mathrm{T}$ and $\mathrm{B}$ cells are activated. Only a tiny fraction of the $\mathrm{B}$ and $\mathrm{T}$ cells passing through the respective $\mathrm{B}$ and $\mathrm{T}$ cell areas become activated, and these are retained in the lymph node, whilst the other unactivated cells can continue to circulate. The 'selected' $\mathrm{B}$ and $\mathrm{T}$ cells, retained in the lymph nodes, then have the maximum chance of finding each other. The selected $\mathrm{T}$ helper cells travel out of the $\mathrm{T}$ cell zone to developing germinal centers within the lymph node, where they can provide help to the selected B cells. As outlined above, this involves cell-cell contact during which the CD40L protein on the $\mathrm{T}$ cell binds to CD40 on the B cell (Fig. 5). The engaged CD40L protein is then rapidly internalized within the T cell, which soon depletes CD40L on the cell surface. This CD40L protein must be replenished on the cell surface, in order for the T cell to continue providing help to B cells, and for this to occur the $\mathrm{T}$ cell must be restimulated. Unfortunately, the T cell is now no-longer in the $\mathrm{T}$ cell zone where the APCs reside that provided the initial stimulation. The solution to this problem is now provided by the B cell. As mentioned above, when the BCR engages the antigen, the antigen is internalized, chopped into fragments, some of which will in turn be presented by MHC class-II molecules on the B cell surface. Once a B cell has been activated it will also express other co-stimulatory proteins (e.g. B7 protein) on its surface (Fig. $5)$. Consequently the B cell can now itself act as an APC and can function to re-stimulate the exhausted T helper cell. So in the lymphoid follicles the $\mathrm{T}$ helper cells and $B$ cells do a 'dance' with each other. The $\mathrm{T}$ helper cell provides the co-stimulation (CD40L) required to activate the $B$ cell, and the $\mathrm{B}$ cell provides the antigen and co-stimulatory molecules $(\mathrm{B} 7)$ required to re-stimulate the $\mathrm{T}$ cell.

The final step in the maturation of a B cell is the choice between becoming a plasma cell or a long-lived membory B cell. The plasma cells are the antibody factories. A 
newly formed plasma cell will usually migrate to the spleen or back to the bone marrow to produce the secreted soluble form of the BCR - the antibody molecule. Plasma cells can produce several thousand antibodies per second, but at the cost of a relatively short life span of only few days.

\section{Linear Peptides as Immunogens?}

Another problem in using synthetic peptides to elicit pathogen specific inhibitory antibodies, is related to their limited ability to mimic epitopes comprising surface patches in a natively folded protein. Linear peptides are inherently flexible, whereas the same regions in folded proteins populate a much more limited region of conformational $(\phi / \psi)$ space. Because short(ish) linear peptides (in the 1980s, the limits of routine solid-phase peptide synthesis were typically around 30-40 residues per chain $^{[58]}$ ) are conformationally flexible in water, there are many ways such a chain can be recognized and bound by an antibody. This might even correlate with the (large) number of different accessible backbone conformations available to the peptide antigen. So it seems likely that only a very small proportion of the antibodies generated in an immune response against a peptide will be able to bind (with comparable affinity) to the same folded sequence in the context of a native protein on the surface of a parasite. A very small proportion of effective neutralizing cross-reactive antibodies in the sera of immunized animals translates into a rather ineffective vaccine.

Today, powerful new methods for protein synthesis and the chemical modification of proteins are available (e.g. the use of pseudo-prolines and other advances in solid-phase synthesis to improve the efficiency of chain assembly; ${ }^{[59-61]}$ native chemical ligation; ${ }^{[62]}$ expressed protein ligation; ${ }^{[63]}$ expanded genetic codes; ${ }^{[64]}$ etc. $\left.{ }^{[65]}\right)$. However, we can add to this a key chemical tool, namely, the possibility to impose conformational constraints on peptide chains, in order to build protein fragments with native-like structures. Introducing conformational constraints in the immunogen, using modified amino acids, templates, crosslinks or other features, should improve the specificity of the immune response for a target epitope in a native protein. In the future, therefore, it seems likely that the tools of conformational stabilization, epitope focusing, and the related idea of scaffold transplantation ${ }^{[66]}$ will have much to contribute to rational vaccine design, although this must go hand-in-hand with an improved understanding of the physical-organic chemical basis of protein recognition in aqueous solution.

\section{Building Synthetic Vaccines}

As mentioned above, key factors contributing to the poor immunogenicity of small synthetic peptides and haptens is the lack of appropriate $\mathrm{T}$ helper epitopes, and their poor capture by APCs. The classical approach to overcome these deficiencies and to increase immunogenicity, is to couple the small molecules to carrier proteins that contain their own $\mathrm{T}$ helper epitopes. However, in many cases the conjugation step is poorly controlled (from a chemical viewpoint) and the conjugates are usually not characterized to determine the effects of conjugation on epitope structure. Moreover, the carrier proteins can introduce their own epitopes that may perhaps be B-cell dominant or even T-cell suppressive. Other carriers have been developed, which address some of these problems, and can be used to generate powerful immune responses against synthetic molecules, when administered together with a strong adjuvant. These carriers include polymers, ${ }^{[67]}$ multiple antigen peptides (MAPs), ${ }^{[68]}$ liposomes $^{[69,70]}$ and immunostimulating complexes (ISCOMs). ${ }^{771]}$

MAPs typically consist of peptide epitopes attached to a branched, dendriticlike, poly-lysine core (Fig. 7). They have been successfully used for experimental vaccination against various infectious diseases, including malaria, schistosomiasis and HIV-1; ${ }^{[72]}$ although this success has not yet been translated into a commercial vaccine product. Both the $\alpha$ - and $\varepsilon$-amino groups in lysine are used to build a branched core, to which both B- and T-cell epitopes can be coupled, and in multiple copies. Such molecules can be synthesised using solid-phase peptide chemistry, and a variety of conjugation methods can be employed to link epitopes or haptens to the poly-lysine core.

Much effort in synthetic vaccine design has gone into the discovery of linear Thelper cell epitopes that are capable of being presented by a broad spectrum of genetically diverse MHC-II molecules; the so-called 'universal' T helper cell epitopes. A number of universal $\mathrm{CD}^{+} \mathrm{T}$ helper epitopes have been identified in proteins from diverse pathogens that bind to a diverse selection of mouse (H-2, IA/IE) and human (HLA, DP/DQ/DR) MHC haplotypes. ${ }^{[73]}$ In at least one case, a broadly applicable artificial $\mathrm{T}$ helper cell epitope (PADRE) has also been designed, based on structural knowledge of how peptides bind to MHC class-II molecules. ${ }^{[74]}$ The molecular basis for the existence of universal $\mathrm{CD}^{+}{ }^{+}$helper epitopes in natural proteins remains incompletely understood. It may be related to their ease of processing and release from antigens by lysosomal or endosomal proteases; ${ }^{[56]}$ to the promiscuous nature of peptide binding by
MHC-II molecules; ${ }^{[75]}$ as well as to limited recognition of MHC-bound peptide by the repertoire of TCRs. ${ }^{[76]}$ It remains an important challenge in synthetic vaccine design to identify and incorporate peptide sequences that can be presented by MHC class-II receptors across a broad spectrum of human and animal haplotypes. Certainly, this is an area where modern peptidomimetic chemistry may also have much to contribute. On the other hand, a drawback in using such universal $\mathrm{T}$ helper epitopes is that they are often distinct from the cognate $\mathrm{T}$ helper epitopes that play a role in the normal physiological response to a pathogen. Where the effector functions of $\mathrm{T}$ cells are important for protection, pathogen-specific $\mathrm{T}$ helper cells will be more valuable with respect to the efficacy of the T cell response than nonrelated peptide epitopes. However, when humoral immunity constitutes an important effector mechanism, a vaccine capable of initiating the production of high concentrations of protective antibodies can nevertheless be very effective, as seen in a malaria vaccine candidate. ${ }^{[77]}$

From the discussion above, the importance in vaccine design of multivalent display of the antigen can be appreciated. Another way to achieve this with small synthetic molecules is to couple them to viruses or virus-like particles (VLPs). VLPs can be grouped into two main classes, based on whether or not there is a cellular membrane (envelope) present. Viral families such as papovaviridae, picornaviride, and parvoviridae are non-enveloped and lipid free (see http://www.virology.net/Big_Virology/ BVHomePage.html). The self-assembling core structures of these natural viruses can be exploited using recombinant DNA technology to display one or more antigens on the surface of the VLPs, simply by inserting a coding sequence at a suitable point in the structural genes. ${ }^{[78]}$ Such non-enveloped VLPs made recombinantly can also be chemically modified, by conjugating antigens to reactive side chain functional groups (e.g. lysine). ${ }^{[79]}$ Other virus families, such as flaviviridae, retroviridae and orthomyxoviridae (influenza) contain a lipid envelope. One such VLP based on an enveloped virus comprises reconstituted influenza viruslike particles, or virosomes. ${ }^{[80]}$ Virosomes are similar to unilammelar liposomes but contain influenza-derived membrane anchored hemagglutinin and neuraminidase (Fig. 8). The presence of the hemagglutinin facilitates cellular uptake and imparts fusogenic activity. Virosomes have been used as carriers to induce specific humoral immune responses against small synthetic peptide antigens, carbohydrates and even synthetic proteins as large as EGF domains, anchored in the viral envelope. ${ }^{[81-86]}$ Upon reconstitution of such VLPs in the presence of peptides conjugated to lipid anchors, the 

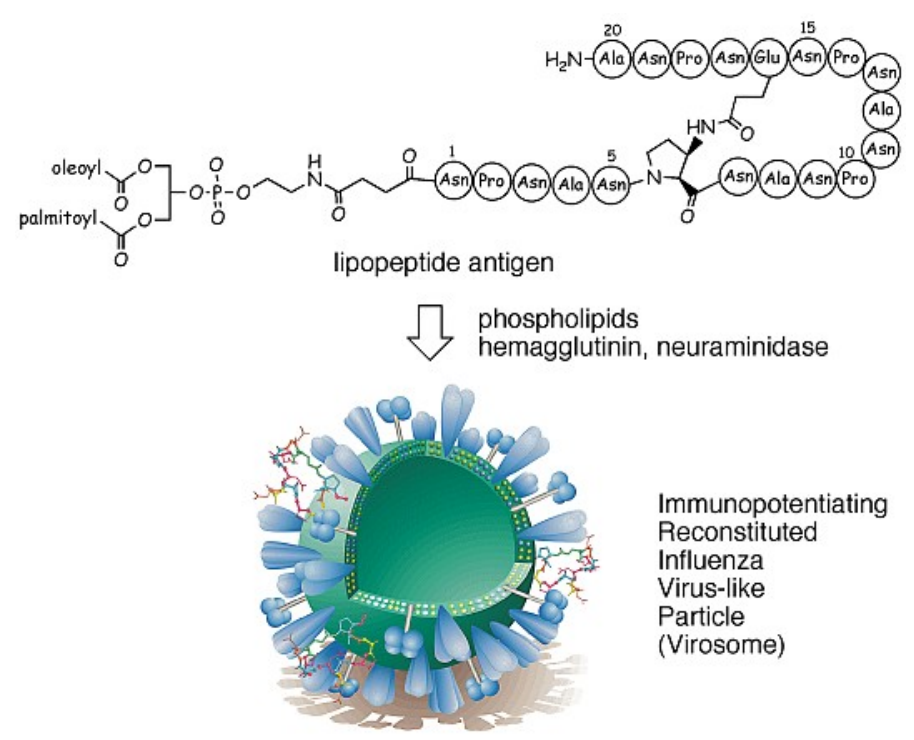

Immunopotentiating

Reconstituted

Influenza

Virus-like

Particle

(Virosome)

Fig. 8. A cartoon representing influenza virus-like particles (virosomes), prepared by self-assembly, from phospholipids (membrane in green) and purified influenza virus glycoproteins (hemagglutinin and neuraminidase, in blue). When phospholipid-linked antigens are included during reconstitution, the antigens can be displayed on the virosomal surface (shown as a stick representation), where they are highly immunogenic.

lipid anchor is inserted in the membrane and the attached synthetic antigen then decorates the surface of the particle, where it can be seen by B cells. The synthetic antigen is then highly immunogenic. Such methods of antigen delivery have proven very effective in generating antigen-specific immune responses without the use of adjuvants. ${ }^{[81-86]}$ Virosomal vaccines against influenza and hepatitis B have been commercially available for several years (Berna Biotech), and over 20 million humans have received virosomal-based vaccines, which have shown an excellent safety record. This clinical experience is very valuable in the development of new vaccines based on synthetic peptides, which use the immunopotentiating activity of virosomes to elicit strong immune responses.

\section{Note added in press}

A new study reported recently in Science (Science 2006, 314, 1936; see also Science 2006, 314, 1859) casts doubt upon the unique importance of TLRs as immune stimulators. Mice lacking the ability to respond to all TLR signals can nevertheless mount impressive antibody responses. Apparently there may be other as yet unknown ways to initiate a strong immune response that do not depend on TLRs.

Received: January 15, 2007

[1] S. A. Plotkin, Nature Med. 2005, 11, S5.

[2] V. W. Bramwell, Y. Perrie, Drug Discov. Today 2005, 10, 1527.

[3] A. Wack, R. Rappuoli, Curr. Opin. Immunol. 2005, 17, 411.
[4] P. H. Lambert, M. Liu, C. A. Siegrist, $\mathrm{Na}$ ture Med. 2005, 11, S54.

[5] B. A. Green, S. M. Baker, Curr. Opin. Microbiol. 2002, 5, 483.

[6] S. G. Reed, A. CamposNeto, Curr. Opin. Immunol. 2003, 15, 456.

[7] D. Serruto, R. Rappuoli, FEBS Lett. 2006, 580, 2985.

[8] M. Mora, D. Veggi, L. Santini, M. Pizza, R. Rappuoli, Drug Discov. Today 2003, 8, 459.

[9] D. R. Burton, Nature Revs. Immunol. 2002, 2, 706. Immunol. 2006, 24, 739.

[11] R. Porter, J. Whelan, in Ciba Foundation Symposium 119, J. Wiley \& Sons, Chichester, UK, 1986.

[12] R. Zinkernagel, Science 1996, 171, 173.

[13] R. Medzhitov, C. A. Janeway, Cell 1997, 91, 295.

[14] A. Pashine, N. M. Valiante, J. B. Ulmer, Nature Med. 2005, 11, S63.

[15] R. J. Ulevitch, Nat. Revs. Immunol. 2004, 4,512 .

[16] P. G. Fallon, A. Alcami, Trends Immunol. 2006, 27, 470.

[17] T. Kawai, S. Akira, Curr. Opin. Immunol. 2005, 17, 338

[18] S. Akira, K. Takeda, Nat. Revs. Immunol. 2004, 4, 499.

[19] B. Beutler, Z. F. Jiang, P. Georgel, K. Crozat, B. Croker, S. Rutschmann, X. Du, K. Hoebe, Annu. Rev. Immunol. 2006, 24, 353.

[20] K. Takeda, T. Kaisho, S. Akira, Annu. Rev. Immunol. 2003, 21, 335.

[21] J. Banchereau, R. M. Steinman, Nature 1998, 392, 245 .
[10] R. Pantophlet, D. R. Burton, Annu. Rev
[22] R. Bonasio, U. H. vonAndrian, Curr. Opin. Immunol. 2006, 18, 503.

[23] B. Breart, P. Bousso, Curr. Opin. Immunol. 2006, 18,483 .

[24] S. Hugues, A. Boissonnas, S. Amigorena, L. Fetler, Curr. Opin. Immunol. 2006, 18 , 491.

[25] J. K. Bell, J. Askins, P. R. Hall, D. R. Davies, D. M. Segal, Proc. Natl. Acad. Sci. USA 2006, 103, 8792.

[26] J. Choe, M. S. Kelker, I. A. Wilson, Science 2005, 309, 581.

[27] E. R. Kandimalla, L. Bhagat, Y. Li, D. Yu, D. Wang, Y.-P. Cong, S. S. Song, J. X. Tang, T. Sullivan, S. Agrawal, Proc. Natl. Acad. Sci. USA 2005, 102, 6925.

[28] S. Agrawal, E. R. Kandimalla, Ann. NY Acad. Sci. 2003, 1002, 30.

[29] D. J. Marciani, Drug Disc. Today 2003, 8, 934.

[30] D. T. O'Hagan, N. M. Valiante, Nat. Revs. Drug Disc. 2003, 2, 727.

[31] J. M. Brewer, Immunol. Lett. 2006, 102, 10.

[32] J. A. Stoute, D. G. Heppner, C. J. Mason, J. Siangla, M. O. Opollo, K. E. Kester, L. Vigneron, G. Voss, M. J. Walter, N. Tornieporth, J. D. Cohen, W. R. Ballou, Amer. J. Trop. Med. Hyg. 2006, 75, 166.

[33] C. R. Kensil, R. Kammer, Exp. Opin. Invest. Drugs 1998, 7, 1475.

[34] T. Okada, J. G. Cyster, Curr. Opin. Immunol. 2006, 18, 278.

[35] J. G. Tew, J. Wu, D. Qin, S. Helm, G. F Burton, A. K. Szakal, Immunol. Rev. 1997, 156, 39.

[36] L. Delamarre, M. Pack, H. Chang, I. Mellman, E. S. Trombetta, Science 2005, 307 1630.

[37] R. M. Zinkernagel, A. LaMarre, A. Ciurea, L. Hunziker, A. F. Ochsenbein, K. D. McCoy, T. Fehr, M. F. Bachmann, U. Kalinke, $\mathrm{H}$. Hengartner, in 'Advances In Immunology', Vol. 79, 2001, pp. 1.

[38] Y. R. Carrasco, F. D. Batista, Curr. Opin. Immunol. 2006, 18, 286.

[39] S. J. Fleire, J. P. Goldman, Y. R. Carrasco, M. Weber, D. Bray, F. D. Batista, Science 2006, 312, 738 .

[40] F. D. Batista, D. Iber, M. S. Neuberger, Nature 2001, 411, 489.

[41] F. D. Batista, M. S. Neuberger, EMBO J. 2000, 19,513 .

[42] A. Grakoui, S. K. Bromley, C. Sumen, M. M. Davis, A. S. Shaw, P. M. Allen, M. L. Dustin, Science 1999, 285, 221.

[43] C. R. F. Monks, B. A. Freiberg, H. Kupfer, N. Sciaky, A. Kupfer, Nature 1998, 395, 82.

[44] C. Wulfing, M. D. Sjaastad, M. M. Davis, Proc. Natl. Acad. Sci. 1998, 95, 6302.

[45] S. Cemerski, A. S. Shaw, Curr. Opin. Immunol. 2006, 18, 298.

[46] T. Saito, T. Yokosuka, Curr. Opin. Immunol. 2006, 18, 305.

[47] R. A. Kroczek, H. W. Mages, A. Hutloff, Curr. Opin. Immunol. 2004, 16, 321.

[48] C. Pasare, R. Medzhitov, Nature 2005, $438,364$. 
[49] C. R. Ruprecht, A. Lanzavecchia, Eur. J. Immunol. 2006, 36, 810.

[50] M. Ghielmetti, A. Reschner, M. Zwicker, E. Padovan, Immunobiology 2005, 210, 211.

[51] G. Jung, K.-H. Wiesmüller, G. Becker, H. J. Buehring, W. G. Bessler, Angew. Chem. Int. Ed. 1985, 10, 872.

[52] F. Reutter, G. Jung, W. Baier, B. Treyer, W. G. Bessler, K.-H. Wiesmüller, J. Pept. Res. 2005, 65, 375 .

[53] W. G. Bessler, L. Heinevetter, K. H. Wiesmuller, G. Jung, W. Baier, M. Huber, A. R. Lorentz, U. V. Desche, K. Mittenbühler, P. Hoffmann, Int. J. Immunopharmac. 1998, 19, 547.

[54] M. Loleit, W. Tröger, K. H. Wiesmuller, G. Jung, M. Strecker, W. G. Bessler, Biol. Chem. Hoppe-Seyler 1990, 371, 967.

[55] C. P. Muller, H. J. Bühring, G. Becker, C. C. Jung, G. Jung, W. Tröger, A. Saalmüller, K. H. Wiesmuller, W. G. Bessler, Clin. Exp. Immunol. 1989, 78, 499.

[56] E. H. Nardin, J. M. Calvo-Calle, G. A. Oliveira, R. S. Nussenzweig, M. Schneider, J.-M. Tiercy, L. Loutan, D. Hochstrasser, K. Rose, J. Immunol. 2001, 166, 481.

[57] J. M. Calvo-Calle, G. A. Oliveira, C. O. Watta, J. Soverow, C. Parra-Lopez, E. H. Nardin, Infect. Immun. 2006, 74, 6929.

[58] R. B. Merrifield, Angew. Chem. Int. Ed. 1985, 24, 799.

[59] F. Albericio, Curr. Opin. Chem. Biol. 2004, 8, 211.

[60] B. L. Bray, Nat. Rev. Drug Discov. 2003, 2, 587.

[61] T. Haack, M. Mutter, Tet. Lett. 1992, 33, 1589.

[62] P. E. Dawson, S. B. H. Kent, Annu. Rev. Biochem. 2000, 69, 923.

[63] T. W. Muir, Annu. Rev. Biochem. 2003, 72, 249.

[64] L. Wang, P. G. Schultz, Angew. Chem. Int. Ed. 2005, 44, 34.

[65] M. E. Hahn, T. W. Muir, Trends Biochem. Sci. 2005, 30, 26.

[66] L. Martin, F. Stricher, D. Missé, F. Sironi, M. Pugnière, P. Barthe, R. Prado-Gotor, I. Freulon, X. Magne, C. Roumestand, A. Ménez, P. Lusso, F. Veas, C. Vita, Nat. Biotech. 2003, 21, 71.

[67] D. C. Jackson, N. O'Brien-Simpson, N. J. Ede, L. E. Brown, Vaccine 1997, 15, 1697.

[68] J. P. Tam, J. Immunol. Meth. 1996, 196, 17.
[69] C. R. Alving, V. Koulchin, G. M. Glenn, M. Rao, Immunol. Rev. 1995, 145, 5.

[70] G. Gregoriadis, I. Gursel, M. Gursel, B. McCormack, J. Control. Release 1996, 41, 49.

[71] I. G. Barr, G. F. Mitchell, Immunol. Cell Biol. 1996, 74, 8.

[72] E. H. Nardin, G. A. Oliveira, J. M. CalvoCalle, R. S. Nussenzweig, Adv. Immunol. 1995, 60, 105.

[73] F. Falugi, R. Petracca, M. Mariani, E. Luzzi, S. Mancianti, V. Carinci, M. L. Melli, O. Finco, A. Wack, A. DiTommaso, M. T. DeMagistris, P. Costantino, G. DelGiudice, S. Abrignani, R. Rappuoli, G. Grandi, Eur. J. Immunol. 2001, 31, 3816.

[74] J. Alexander, J. Sidney, S. Southwood, J. Ruppert, C. Oseroff, A. Maewal, K. Snoke, H. M. Serra, R. T. Kubo, A. Sette, H. M. Grey, Immunity 1994, 1, 751.

[75] D. A. Mason, Immunol. Today 1998, 19 , 395.

[76] M. G. Rudolph, R. L. Stanfield, I. A. Wilson, Annu. Rev. Immunol. 2006, 24, 419.

[77] H. M. Etlinger, D. Gillessen, H.-W. Lahm, H. Matile, H.-J. Schönfeld, A. Trzeciak, Science 1990, 249, 423.

[78] L. X. Doan, M. Li, C. Y. Chen, Q. Z. Yao, Rev. Med. Virol. 2005, 15, 75.

[79] R. L. Garcea, L. Gissmann, Curr. Opin. Biotechnol. 2004, 15, 513.

[80] R. Zurbriggen, Vaccine 2003, 21, 921.

[81] B. Pfeiffer, E. Peduzzi, K. Moehle, R. Zurbriggen, R. Glück, G. Pluschke, J. A. Robinson, Angew. Chem. Int. Ed. 2003, 42, 2368.

[82] R. Moreno, L. Jiang, K. Moehle, R. Zurbriggen, R. Glück, J. A. Robinson, G. Pluschke, ChemBioChem 2001, 2, 838.

[83] F. Pöltl-Frank, R. Zurbriggen, A. Helg, F. Stuart, J. A. Robinson, R. Glück, G. Pluschke, Clin. Exp. Immunol. 1999, 117, 496.

[84] M. S. Müller, A. Renard, F. Boato, D. Vogel, M. Naegeli, R. Zurbriggen, J. A. Robinson, G. Pluschke, Infect. Immun. 2003, $71,4749$.

[85] S. James, K. Moehle, A. Renard, M. S. Mueller, D. Vogel, R. Zurbriggen, G. Pluschke, J. A. Robinson, ChemBioChem 2006, 7, 1943.

[86] X. Y. Liu, S. Siegrist, M. Amacker, R. Zurbriggen, G. Pluschke, P. H. Seeberger, ACS Chem. Biol. 2006, 1, 161. 